\title{
Weniger Schlaganfälle in den letzten Jahren
}

Fragestellung: Wie hoch ist das Risiko, innerhalb eines Jahres nach einer transienten ischämischen Attacke (TIA) oder einem leichten Schlaganfall, einen Schlaganfall zu erleiden?

Hintergrund: Eine Reihe von Studien, die zwischen 1997 und 2003 durchgeführt wurden, schätzte das Risiko eines Schlaganfalls oder eines akuten Koronarsyndroms in den ersten drei Monaten nach einer TIA oder einem leichten Schlaganfall auf $12-20 \%$. Damals standen allerdings viele therapeutische Maßnahmen, wie Behandlung auf der Stroke Unit, rascher Beginn einer wirksamen antithrombotischen Therapie, eine ausreichende antihypertensive Behandlung und eine Prophylaxe mit Statinen nicht zur Verfügung. Daher war es sinnvoll, in einer neuen prospektiven Studie zu untersuchen, ob sich seit dem Jahr 2000 die Prognose von Patienten mit TIA oder leichtem Schlaganfall verbessert hat.

Patienten und Methodik: Die multinationale Studie, die auch deutsche Studienzentren hatte, rekrutierte Patienten mit TIA oder leichtem Schlaganfall innerhalb der vorausgegangenen sieben Tage. Berechnet wurden das 1-Jahres-Schlaganfallrisiko und der kombinierte Endpunkt von Schlaganfall, akutem Koronarsyndrom oder Tod durch kardiovaskuläre Ursachen. Es wurde außerdem eine Validierung des $\mathrm{ABCD}^{2}$-Scores vorgenommen, der zwischen 0 und 7 liegt. Bei diesem Score steht A für Alter, B für erhöhte Blutdruckwerte, $C$ für die klinischen Symptome und D für die Dauer der Symptomatik. Außerdem wurde die Rezidivrate mit den Ergebnissen der zerebralen Bildgebung und mit der Ätiologie von TIA und leichAmarenco P, Lavallée PC, Labreuche $J$ et al. One-year risk of stroke after transient ischemic attack or minor stroke. N Engl J Med 2016; 374: 1533-42 tem Schlaganfall korreliert.

Ergebnisse: Zwischen 2009 und 2011 wurden $4.789 \mathrm{~Pa}$ tienten in 61 Studienzentren in 21 Ländern rekrutiert. Fast

\section{- Kommentar von Hans-Christoph Diener, Essen \\ Beruhigendes Ergebnis}

Diese große Registerstudie ist sehr beruhigend, da sie zeigt, dass sich ganz offenbar die frühe und anhaltende Sekundärprävention des Schlaganfalls bei Patienten mit TIA und leichtem Schlaganfall positiv auswirkt. Während die durchschnittliche Schlaganfallrate vor 15 Jahren noch zwischen $12 \%$ und $15 \%$ pro Jahr betrug, ist sie jetzt auf $5 \%$ abgefallen. Für den klinischen Alltag sind die Prädiktoren für einen erneuten Schlaganfall relevant, da sie ermöglichen, die Patienten zu identifizieren, die besonders aggressiv behandelt werden müssen und bei denen sich eine strukturierte Nachsorge lohnen würde. Entsprechende Präventionsprogramme existieren allerdings in strukturierter Form in Deutschland nur in sehr eingeschränktem Umfang.

$80 \%$ der Patienten wurden innerhalb von 24 Stunden nach Symptombeginn untersucht. In der Bildgebung hatten $33 \%$ eine akute zerebrale Ischämie. $23 \%$ hatten eine Makroangiopathie mit einer über 50 \%igen Stenose der hirnversorgenden Arterien. 10,4\% der Patienten hatten Vorhofflimmern. Das 1-Jahres-Risiko des kombinierten kardiovaskulären Endpunktes lag bei 6,2\% mit einem 95\%-Konfidenzintervall von 5,5-7,0. Die Schlaganfallraten betrugen 1,5\% für den Tag 2, 2,1 \% für den Tag 7, $2,8 \%$ für den Tag 30 und 3,7\% für den Tag 90 sowie 3,7\% und $5,1 \%$ nach einem Jahr. Prädiktoren für einen erneuten Schlaganfall waren multiple Infarkte in der zerebralen Bildgebung, eine Makroangiopathie und ein $\mathrm{ABCD}^{2}$-Score von 6 oder 7.

Schlussfolgerungen: Im Vergleich zu dem Zeitraum 1997 bis 2003 ist zwischen 2009 und 2011 die vaskuläre Ereignisrate bei Patienten mit TIA und leichtem Schlaganfall deutlich geringer. Mithilfe des $\mathrm{ABCD}^{2}$-Scores, der pathogenetischen Einordnung und der zerebralen Bildgebung können Patienten mit erhöhtem Risiko identifiziert werden, die eine besonders aggressive konservative Therapie benötigen.

\section{Erratum}

Erratum zum Beitrag: Internetbasierte Interventionen als supportive Therapie bei Depressionen. Neuer Baustein für die antidepressive Therapie?

David Herzog

InFo Neurologie \& Psychiatire

November 2016, Volume 18, Issue 11, pp 12

DOI: 10.1007/s15005-016-1947-6

Date: 23 Nov 2016

(c) Springer Medizin Verlag $\mathrm{GmbH}$, Berlin

Der zweite Satz des Kommentars wird ersatzlos gestrichen. Der Kommentar lautet richtig:

Für eine generelle Therapieempfehlung ist es noch zu früh Die vorliegende randomisierte, kontrollierte, einfach verblindete, prospektive, multizentrische Studie glänzt mit guter Studienqualität, einer großen Fallzahl und einem durchdachten naturalistischen Studiendesign, unabhängig von kategorialen Diagnosen. Außerdem konnte aufgrund des Studiendesigns keine doppelte Verblindung durchgeführt werden, was die Aussagekraft der Ergebnisse schmälert. Dies ist vor allem deshalb relevant, da die ermittelten Effektstärken eher im niedrigen Bereich anzusiedeln sind, und daher eigentlich eine möglichst hohe Studienqualität nötig ist, um probate Aussagen tätigen zu können. In Anbetracht verschiedener Vorstudien mit zum Teil konträren oder unzureichend statistisch untermauerten Ergebnissen bringt die vorliegende Studie einen großen Zugewinn. Hieraus eine generelle Therapieempfehlung für Patienten mit leichten bis mittelschweren depressiven Symptomen - wie die Autoren der Studie dies tun abzuleiten, wäre allerdings zu früh, denn eine weitergehende intensive Beschäftigung mit diesem Thema, inklusive einer Kosten-Nutzen-Analyse, ist erforderlich.

Zitierweise: Herzog D. Internetbasierte Interventionen als supportive Therapie bei Depressionen. Neuer Baustein für die antidepressive Therapie? InFo Neurologie. 2017; 19 (1): 31. DOI: 10.1007/s15005-017-2007-6 